\title{
When visual and verbal memories compete: Evidence of cross-domain limits in working memory
}

\author{
CANDICE C. MOREY and NELSON COWAN \\ University of Missouri, Columbia, Missouri
}

\begin{abstract}
Recently, investigators have suggested that visual working memory operates in a manner unaffected by the retention of verbal material. We question that conclusion on the basis of a simple dual-task experiment designed to rule out phonological memory and to identify a more central faculty as the source of a shared limitation. With a visual working memory task in which two arrays of color squares were to be compared, performance was unaffected by concurrent recitation of a two-digit list or a known seven-digitsequence. However, visual working memory performance decreased markedly when paired with a load of seven random digits. This was not a simple tradeoff, inasmuch as errors on the visual array and high digit load tasks tended to co-occur. Working memory for digits and visual information thus are both subject to at least one type of shared limit, not just domain-specific limitations. The nature of the shared limit is discussed.
\end{abstract}

Working memory, the temporary retention of limited information in an accessible form, underlies various mental processes, including perceptual comparison, language comprehension, and calculation (Baddeley, 1986). Lately, the domain specificity of working memory has become a controversial issue. The same is true of a human faculty that is an important part of working memory: selective attention (Cowan, 1995). Recent studies have suggested that there is no interference between working memory tests in the visuospatial and verbal domains and, therefore, no reliance on a common attentional resource (Cocchini, Logie, Della Sala, MacPherson, \& Baddeley, 2002; Luck \& Vogel, 1997; Scholl \& Xu, 2001). It also has been suggested that each feature map in memory has its own separate capacity limit (Wheeler \& Treisman, 2002), that attention has a capacity that is restricted within, but not between, sensory modalities (Duncan, Martens, \& Ward, 1997), and that perception and working memory use resources different from those that underlie response selection and execution (Wickens, 2002).

Against this sentiment, some authors have suggested that there is a fundamental limit that holds across different domains, such as a limit in the concurrent contents of the focus of attention (Cowan, 2001), in the capacity of an episodic memory buffer (Baddeley, 2001), in central processing (Arnell \& Jolicœur, 1999; Jolicœur, 1999;

This research was supported by NIH Grant R01 HD-21338 awarded to N.C. We thank J. Scott Saults for making digit stimuli and providing helpful comments and Derek Besner, Pierre Jolicœur, Jeff Rouder, and Eric Ruthruff for comments on earlier drafts. Address correspondence to C. C. Morey or N. Cowan, Department of Psychological Sciences, University of Missouri, 207 McAlester Hall, Columbia, MO 65211 (e-mail: clcfyb@mizzou.edu [C.C.M.] or cowann@missouri.edu [N.C.]).
Tombu \& Jolicœur, 2003), or in the use of a common mechanism in working memory and attention tasks (Cowan, 1988, 1995; de Fockert, Rees, Frith, \& Lavie, 2001). These central limits imply that one should be able to show interference between, for example, visuospatial and verbal working memory tasks.

A straightforward means of distinguishing between these views is to examine the amount of interference between two dissimilar working memory tasks. For example, Cocchini et al. (2002) compared accuracy in singletask and dual-task procedures by embedding one task in another (e.g., hear digit sequence, examine spatial pattern, recall spatial pattern, and recall digit sequence). They found small effects of dual-task load on recall of the inner task but argued that much larger effects would be expected if there were true reliance on a resource common to the two tasks. We consider the outcome of this study ambiguous. One limitation is that it did not separate dual-task performance into trials according to accuracy on the outer task (the task imposing a memory load). When one task was verbal and one was nonverbal, participants responded correctly to the outer task about $80 \%-$ $90 \%$ of the time, which may mean that the tasks were too easy to tax a common resource fully.

We addressed this question with a simple and elegant visuospatial working memory task, the visual array comparison task of Luck and Vogel (1997). In this task, a spatial array of color squares (sample array) is followed by a second array (test array) that either is identical to the first or differs in the color of one square. A same or different response is required. Because it is possible for a given array to contain multiple squares of the same color, the task requires memory for the correspondence or binding between the colors and the locations of the squares. 
In the version of the task that we employed, there was a circle surrounding one square in the test array, and if anything changed between arrays, it was the color of the encircled square. This version of the task is advantageous because only one decision must be made. Performance levels on this sort of task are very high with up to four squares per array but decrease rapidly as array size increases.

According to the most widely discussed theory of working memory (Baddeley, 1986), there are two main ways in which the visual array task could suffer interference from concurrent verbal stimuli. First, participants could use verbal coding to remember some of the colors (e.g., "the top left square is red"). Second, both visuospatial and auditory tasks could require a mental faculty that is used for retention of stimuli from any domain. This mental faculty could be the manipulation of information by central executive processes (Baddeley, 1986) or the entry of some stimulus information from each domain into the focus of attention, either for storage (Cowan, 2001) or for transfer to another storage medium, such as the episodic buffer (Baddeley, 2000).

Luck and Vogel (1997) controlled for verbal coding of visual arrays. On trials in which they presented two digits before the sample array and tested memory for the digits after the response to the test array, visual performance was no different from that in the control condition, in which there was no digit load. The presumption was that it was not possible to carry on verbal coding of the arrays and still execute the digit task. The absence of an effect of the digit task was as expected, because the brevity of the sample array was thought to discourage verbal coding in the first place, although such coding cannot be ruled out entirely even with a concurrent load.

However, this manipulation leaves several uncertainties. First, given that the load was to be held silently, it is possible that it could be memorized and, therefore, would not actually require ongoing use of verbal working memory during the visual array comparison task. Second, and more interesting, a two-digit load is not suitable for examining the possibility that a cross-domain faculty is shared between visual and verbal working memory. Previous work has shown that a digit load must include about six digits before interference effects can be observed (Baddeley \& Hitch, 1974).

Cowan (2001) suggested that the visual array comparison task of Luck and Vogel (1997) may be a measure of the capacity of the focus of attention. The rationale was that the second array overwrites the visual memory of the first array before a decision can be made, so that a low-level visual form of memory would not be useful in this task. Instead, information must be extracted from the visual array into a more abstract form that can survive the presentation of the second array. Cowan (2001) suggested that attention is that storage medium. According to this theoretical interpretation of the task, visuospatial memory should be susceptible to interference from verbal materials when they are increased beyond the point at which they can be recalled through passive verbal rehearsal alone. People can recall about as much verbal material as they can recite in about $2 \mathrm{sec}$ (Baddeley, 1986). Beyond the amount that can be rehearsed, though, they could use central executive processes and the focus of attention to try to maintain additional verbal information.

In our experiment, the articulation and load aspects of a secondary task were investigated together. On different trials, different types of spoken stimuli were presented before the first visual array: a two-digit memory load, a seven-digit memory load, reference to the participant's own seven-digit telephone number, or the phrase "nothing to say." One's telephone number is the same length as the seven-digit load but has been memorized and, therefore, is assumed to impose little or no load on working memory. Except in the case of "nothing to say," the digits were to be recited aloud repeatedly throughout the visual array task. Assuming that vocal recitation inhibits use of the verbal coding and rehearsal mechanism (Baddeley, 1986), if visual arrays are retained partly through verbal coding, there should be interference between verbal and visual tasks in all three recitation conditions. If visual array storage relies on cross-domain resources (e.g., attention), there should be interference only in the seven-digit-load condition. If there is no such reliance on a general form of storage, there might be no interference between tasks at all.

It is also possible to predict the form of the change in visual array performance as a function of the auditory memory load. Cowan (2001) described a formula to estimate $k$, the number of squares from the sample array retained in working memory at the time that the test array is presented. A modification of a formula offered by Pashler (1988), Cowan's (2001) formula assumes that if the square in the sample array corresponding to the cued square in the test array is retained in working memory, the participant will answer correctly, whether there has been a change (leading to a hit) or not (leading to a correct rejection). If the square is not retained in working memory, the participant will guess different at some rate $g .{ }^{1}$ If a memory load reduces $k$ by a fixed amount across array sizes, this turns out to produce a greater reduction in the proportion correct for smaller arrays. In contrast, the absence of this sort of interaction would suggest that the auditory memory load operates in some manner other than by occupying a slot in working memory.

\section{METHOD}

\section{Design}

Figure 1 shows a trial's stimulus arrangement. In a 4 (auditory stimulus conditions) $\times 3$ (visual array sizes) $\times 2$ (array change vs. no change) within-subjects design, the 24 conditions were randomly mixed in each stimulus block. The four auditory conditions were (1) no digit load (i.e., "nothing to say"), (2) a two-digit load to be recited aloud repeatedly throughout the trial until the response to the visual array, at a rate of about 3 digits/sec, (3) a seven-digit load to be recited in that manner, and (4) recitation in that manner 


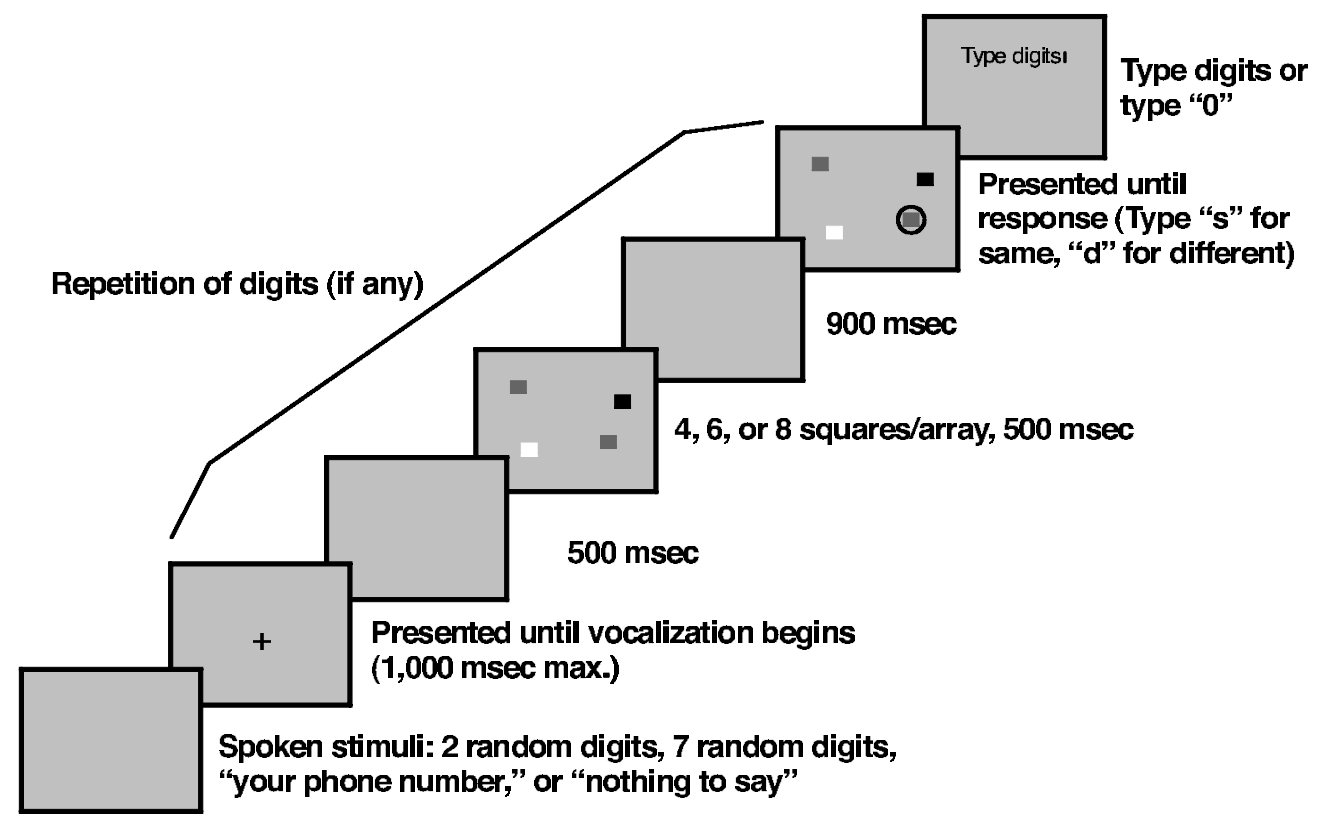

Figure 1. Experimental design. Conditions were randomly intermixed at presentation. Colors of squares (depicted here by shades of gray) were chosen randomly with replacement from seven discriminable colors (i.e., it was possible for an array to contain multiple squares of the same color).

of the participant's own seven-digit telephone number. The two visual arrays included four, six, or eight squares each. The experiment began with 6 practice trials, one for each condition and then an extra seven-digit trial and an extra "nothing to say" trial. The practice trials were followed by 168 test trials (seven blocks of a complete set of 24 trial types, randomly mixed).

\section{Participants}

The participants were 24 undergraduates ( 16 females and 8 males), between 18 and 23 years old, completing course requirements.

\section{Apparatus and Stimuli}

The auditory stimuli were digitally stored and presented via audio (TDH-49 type) headphones. Each list of digits, spoken in a male voice, was drawn randomly from the digits 1-9 without replacement. Each individual digit had been recorded to fit within a window of $500 \mathrm{msec}$ and occupied the beginning of that window. There also were trials in which, instead of digit lists, the phrase "your phone number" or "nothing to say" was presented in a female voice. The auditory presentations fell between 74 and $80 \mathrm{~dB}(\mathrm{~A})$, as measured with a sound level meter and earphone coupler.

A voice key was used to present visual stimuli at a fixed time relative to the onset of the participants' vocalizations. Visual stimuli were displayed on a 17-in. color monitor at a viewing distance of about $50 \mathrm{~cm}$. The visual arrays included four, six, or eight squares $\left(0.65^{\circ} \times 0.65^{\circ}\right)$ arranged randomly on a neutral gray background, each with a color randomly selected from one of seven easily discriminable colors (red, blue, violet, green, yellow, black, or white). The items in the arrays were separated by at least $2^{\circ}$ of visual angle, measured from the centers of the squares. Two arrays (sample and test arrays) were presented on every trial, and a single color square within each test array was surrounded by a black circle. The squares of the sample and the test arrays either were exactly the same in both color and location or differed only in the color of the encircled square.

\section{Procedure}

Each participant was tested in an isolated quiet booth. The session began with instructions. The participant initiated the trial with a keypress and then heard the auditory memory instructions (a twoor seven-digit list or the phrase "your phone number" or "nothing to say"). A fixation cross immediately appeared and was removed when the voice key detected the beginning of the participant's vocalization. Activation of the voice key started the visual presentation, beginning with a 500-msec blank interval, followed by the onset of the sample array. If vocalization did not begin within $1 \mathrm{sec}$ of the onset of the fixation cross, the trial was reinitiated (with the same trial type) to avoid a lengthy unfilled period during which the digit load could be memorized. In the control condition ("nothing to say"), the fixation cross lasted $1 \mathrm{sec}$ and was followed by the same visual events as those in the other three conditions.

The sample array lasted for $500 \mathrm{msec}$ and was followed by a 900msec blank interval and then the test array with one square encircled. When the test array occurred, the participant was to stop articulating and respond to the array as quickly and accurately as possible. The test array remained on the screen until the participant pressed one of the appropriate keys ("s" for when the probed square was the same color as previously, " $d$ " for when it was different). Immediately afterward, the digit load was to be typed into the computer from memory, followed by the return key when the participant was satisfied with the answer. In the control and telephone conditions, the participants were to enter a " 0 ." An experimenter listened outside of the testing booth to ensure that the participants repeated the digits (if required) until they responded to the test array.

\section{RESULTS}

Loads of two and seven digits were correctly recited without error on $98 \%$ and $45 \%$ of the trials, respectively. Figure 2 shows the percentage of correct visual array comparisons for arrays of four, six, and eight squares ( $x$-axis) under each recital condition (graph parameter). An analy sis of variance (ANOVA) of the visual task proportion correct, with recital condition and visual array size as factors (excluding trials with digit recall errors 


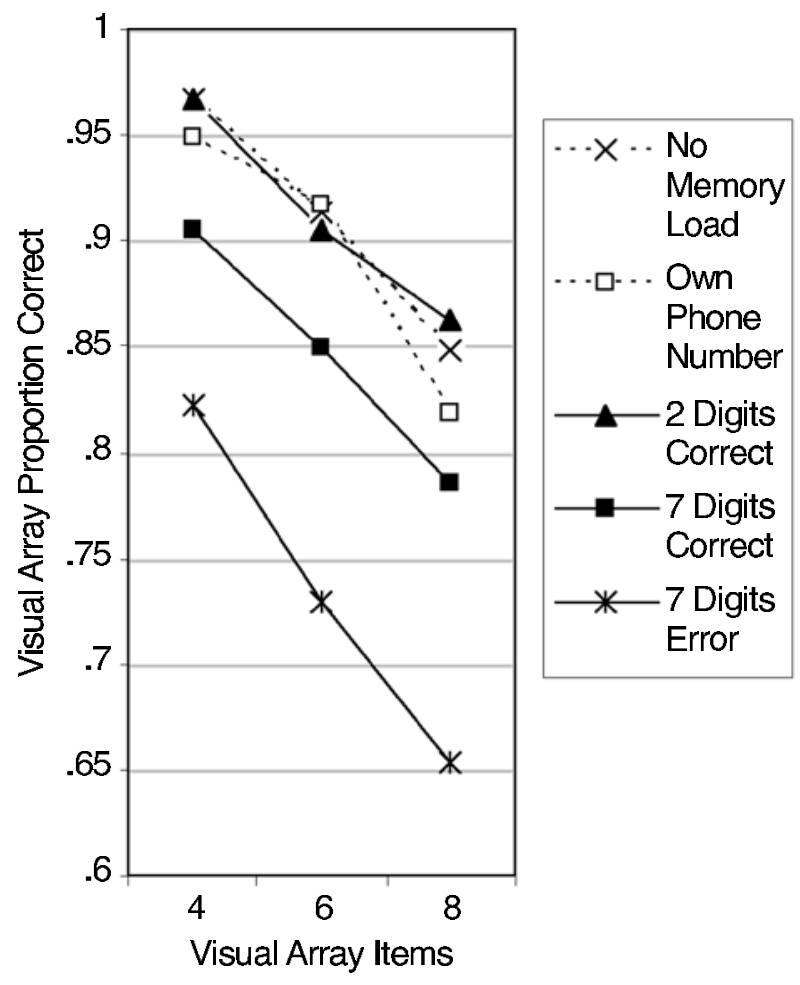

Figure 2. Proportion correct in comparisons of two arrays that were identical or different in the color of a single square, as a function of the number of visual items in the array ( $x$-axis) and the recitation task that was carried out concurrently with the visual array comparison task (graph parameter). A seven-digit memory load impaired visual array comparisons.

and, thus, excluding two participants from the analysis who had no data in some cells), produced statistically significant effects of recital condition $[F(3,63)=7.54$, $p<.001]$ and visual array size $[F(2,42)=24.90, p<$ $.001]$, but no interaction $(F<1)$. Post hoc Newman-Keuls tests indicated that array comparison performance with a seven-digit load ( $85 \%$ correct) was poorer than that with a two-digit load (92\%), a telephone number $(90 \%)$, or no load (91\%; SEM $\leq 2 \%$ in each case). The latter three conditions did not differ.

The absence of an interaction of recital condition and visual array size cannot be attributed to the rather high performance of some participants on the task. When a median split on visual array performance was carried out, separate ANOVAs for participants above and below the median both showed nonsignificant interactions ( $F<1$ in both cases).

When the seven digits were recalled incorrectly (Figure 2, bottom line), visual array performance was much lower $(74 \%$ correct, $S E M=3 \%)$. For 21 participants with both correct and incorrect seven-digit recalls at each set size (necessary for a fair statistical test), visual array comparisons were significantly more accurate when accompanied by correct list recall $(84 \%)$ than when accompanied by incorrect list recall $[72 \% ; F(1,20)=$
$16.05, p<.001]$. The main effect of array size was again significant but did not interact with list recall accuracy.

We examined auditory digit list memory performance, taking into consideration the influence of visual task conditions and responses. We examined this among 16 participants who had both correct and incorrect visual array responses on some trials with every array size. Although there was no significant effect of visual array size on the percentage of trials with correct seven-digit recall $(F<1)$, a higher percentage of seven-digit recalls was correct on trials in which responses to visual arrays were correct $(\mathrm{M}=48 \%, S E M=5 \%)$ than on trials in which responses to visual arrays were incorrect $[M=33 \%$, $S E M=6 \% ; F(1,15)=9.09, p<.01]$. The factors of visual array size and visual response accuracy did not interact in predicting digit list recall $(F<1)$. Across all 24 participants, all of whom had trials with correct visual array performance at each array size, it was not the case that digits were less often recalled correctly as visual array size increased. With array sizes of four, six, and eight, correct digit recall occurred on an average of $.46, .48$, and .52 of such trials, respectively ( $S E M=.10$ in each case). This suggests that participants do not abandon digit recall when the visual array task becomes difficult.

The positive relation between visual and auditory task performance on a trial-by-trial basis cannot be attributed to improvement in both tasks over time. An examination of performance in both modalities across seven trial blocks revealed no interaction of modality and trial block $\left[F(6,138)=1.18, M S_{\mathrm{e}}=0.03, p=.32\right]$. Also, an inspection of a scatterplot of auditory versus visual performance revealed no individuals who were outliers on both tasks.

\section{DISCUSSION}

Luck and Vogel (1997) imposed only a two-digit memory load and found no effect upon visual array comparisons. We replicated that effect, using vocal recitation of the two-digit load, ensuring that the load did, as was assumed, suppress verbal coding of the arrays (cf. Baddeley, 1986). No differences were observed between outcomes in the two-digit-load, no-suppression, and participant's own telephone number recitation conditions. However, performance was lower with a memory load of seven random digits. Moreover, the present study may be the first to demonstrate that the amount of dual-task interference can be much greater when performance on the load task (here, the digit list task) is inaccurate (a 19\% effect on visual array performance) than when it is accurate $(7 \%$ effect).

Our results counter the claim (Luck \& Vogel, 1997; Vogel, Woodman, \& Luck, 2001) that array comparisons can be carried out on the basis of a modality-specific visual memory, without a central resource. Although verbal coding was apparently not important for the visual array task, given the absence of an effect of a memorized seven-digit recitation (the participant's telephone num- 
ber), the tasks appeared to share a central resource of working memory, given the effects of a novel seven-digit memory load.

Cocchini et al. (2002) emphasized that they observed "concurrent performance of two memory tasks." However, we see no actual conflict between their results and ours. Like us, they obtained a significant effect of a digit preload on a visual pattern memory task. Their effect magnitude in two experiments, $4 \%-5 \%$, is similar to what we obtained on trials in which the digit load was recited correctly. However, they did not separately report trials in which the digit preload was recited incorrectly. Moreover, they used a load short enough that it was recited correctly about $80 \%$ of the time or more when combined with pattern recall. Had they separated visual performance according to correct or incorrect digit list recall, they might have found, as we did, that visual array performance is affected by a digit preload much more on trials in which the preload is incorrectly recalled. We found array comparison performance levels to be about $16 \%$ lower with a load incorrectly recalled than with no load. These results contradict a noninterference hypothesis.

The magnitude of a common holding place for digits and color squares can be estimated by employing the measure of capacity (Cowan, 2001) discussed above. With that measure, the $85 \%$ correct performance that accompanied correct seven-digit recall corresponded to $k=4.2$ squares, whereas the $90 \%$ correct performance that accompanied recital of the participant's own telephone number corresponded to $k=4.8$ squares. In the other two conditions, similarly, $k=4.9$ and 5.0 squares. Thus, the cost of a correctly remembered seven-digit load was only $0.6-0.8$ square. For trials in which a seven-digit load was not correctly recalled, for which visual array comparisons were at $74 \%$ correct, visual capacity could be estimated at 2.9 color squares, about 2 squares less than with no load.

The fact that seven digits cost less than 1 square in memory poses some difficulty for the notion that both types of materials are held primarily in a common workspace. If digits and color squares were held exclusively in a common workspace, one would expect that each digit recalled would diminish visual array capacity by 1 color square (under the assumption that each color or digit is a separate chunk in memory) or about 0.5 color square (under the assumption that seven digits typically are combined into about 3.5 chunks, whereas random squares are not chunked; see Cowan, 2001). Also, as was explained in the introduction, the absence of an interaction between visual array size and the load condition (see Figure 2) is inconsistent with a mechanism in which a seven-digit load reduces visual capacity by a fixed number of squares regardless of array size. Instead, capacity is reduced more at larger array sizes.

There appear to be several plausible theoretical accounts of the results. First, it is possible that there is a common workspace but that it was not drawn upon heavily in the present study. The digit load might have been held primarily in a phonological form, and attention might have been used just to supplement phonological memory. Attention could be needed either for the best possible covert rehearsal or to directly retain the "overflow" of digits that do not fit into the phonological rehearsal cycle. Either mechanism is consistent with the existence of a common workspace, such as the focus of attention (Cowan, 2001). The assumption would be that the present dual-task design was not ideal for demonstrating that common workspace, because the digit task did not load the workspace heavily enough.

A second interesting possibility is that there is no common workspace. This is not necessarily inconsistent with the notion that a limitation in the capacity of attention restricts the amount retrieved from any stimulus set to about four independent chunks (Cowan, 2001). However, the limit might apply only at the point at which information is transferred from a peripheral or sensory representation to a more abstract representation. According to this account, in our procedure, when attention was diverted from the visual modality during and/or slightly after the presentation of the sample array, fewer color squares would be represented in visual working memory. Once the transfer of color squares to visual working memory was complete, its retention would be immune to further distraction (provided that the retention interval is relatively short, as in our 900-msec interval).

Wheeler and Treisman (2002) presented visual arrays with multiple features per object and found an ability to retain multiple types of features, along with a limitation in how many instances of each feature type could be retained. They proposed that "feature values from different dimensions are each stored in parallel in their own dimensionspecific cache, within which feature values compete for limited capacity representation but between which there is little or no competition" (p. 61). However, as has been indicated above, another explanation is that the limitation in performance occurs in what can be transferred to a central attentional resource when it focuses on any one feature map.

The most straightforward interpretation of what Cowan (2001) has said about capacity limits is that attention itself serves as the holding device. The alternative mentioned above, that the attentional limit just determines how much information can be transferred to a holding device that has no capacity limit itself, seems more consistent with one version of the theory proposed by Baddeley (2000). That article did not clarify the capacity limits of the episodic buffer, although a capacity limit was suggested by Baddeley (2001). However, it seems plausible that an episodic buffer could be a holding device without a capacity limit itself, limited only by the amount of attention available to transfer information into that buffer. At present, we cannot distinguish between the theoretical possibilities of a capacity limitation in storage or just in information transfer.

In sum, this article provides a qualification of other recent studies that have left the impression that there is lit- 
tle conflict between working memory in different domains (Cocchini et al., 2002; Luck \& Vogel, 1997; Scholl \& $\mathrm{Xu}, 2001)$. Specifically, when we separated trials according to correct versus incorrect performance on each task, we found that incorrect retention of a digit preload often was accompanied by incorrect visual array comparison performance and vice versa. The findings helped to generate a new hypothesis that synthesizes the concept of an attention-related limitation in working memory capacity (Cowan, 2001) and the possible storage of information without a capacity limit. More research is needed to distinguish among hypotheses, because the mechanisms used to retain any particular stimulus in working memory are typically open to debate, as are the interpretations of dual-task results (Luck \& Vecera, 2002; Ruthruff \& Pashler, 2001).

\section{REFERENCES}

ARNELL, K. M., \& JOLICÄUR, P. (1999). The attentional blink across stimulus modalities: Evidence for central processing limitations. Journal of Experimental Psychology: Human Perception \& Performance, 25, 630-648.

BADDELEY, A. D. (1986). Working memory. Oxford: Oxford University Press, Clarendon Press.

BADdELEy, A. D. (2000). The episodic buffer: A new component of working memory? Trends in Cognitive Sciences, 4, 417-423.

BADDELEY, A. D. (2001). The magic number and the episodic buffer. Behavioral \& Brain Sciences, 24, 117-118.

BAdDeley, A. D., \& Hitch, G. J. (1974). Working memory. In G. A. Bower (Ed.), The psychology of learning and motivation (Vol. 8, pp. 47-89). New York: Academic Press.

Cocchini, G., Logie, R. H., Della Sala, S., MacPherson, S. E., \& BADDELEY, A. D. (2002). Concurrent performance of two memory tasks: Evidence for domain-specific working memory systems. Memory \& Cognition, 30, 1086-1095.

CoWAN, N. (1988). Evolving conceptions of memory storage, selective attention, and their mutual constraints within the human information processing system. Psychological Bulletin, 104, 163-191.

CoWAN, N. (1995). Attention and memory: An integrated framework. New York: Oxford University Press.

CowAN, N. (2001). The magical number four in short-term memory: A reconsideration of mental storage capacity. Behavioral \& Brain Sciences, 24, 87-114.

De Fockert, J. W., Rees, G., Frith, C. D., \& Lavie, N. (2001). The role of working memory in visual selective attention. Science, 291, 1803 1806.
Duncan, J., MARTENS, S., \& WARD, R. (1997). Restricted attentional capacity within but not between sensory modalities. Nature, $\mathbf{3 8 7}$, 808-810.

JOLICÄUR, P. (1999). Restricted attentional capacity between sensory modalities. Psychonomic Bulletin \& Review, 6, 87-92.

LuCK, S. J., \& VECERA, S. P. (2002). Attention. In H. Pashler \& S. Yantis (Eds.), Stevens' Handbook of experimental psychology (3rd ed., pp. 235-286). New York: Wiley.

LUCK, S. J., \& VogeL, E. K. (1997). The capacity of visual working memory for features and conjunctions. Nature, 390, 279-281.

PASHler, H. (1988). Familiarity and visual change detection. Perception \& Psychophysics, 44, 369-378.

Ruthruff, E., \& PAShler, H. E. (2001). Perceptual and central interference in dual-task performance. In K. Shapiro (Ed.), The limits of attention: Temporal constraints in human information processing (pp. 100-123). New York: Oxford University Press.

SCHOLL, B. J., \& XU, Y. (2001). The magical number 4 in vision. Behavioral \& Brain Sciences, 24, 145-146.

TOMBU, M., \& JOLICÄUR, P. (2003). A central capacity sharing model of dual-task performance. Journal of Experimental Psychology: Human Perception \& Performance, 29, 3-18.

Vogel, E. K., Woodman, G. F., \& Luck, S. J. (2001). Storage of features, conjunctions, and objects in visual working memory. Journal of Experimental Psychology: Human Perception \& Performance, 27, 92-114.

Wheeler, M. E., \& Treisman, A. M. (2002). Binding in short-term visual memory. Journal of Experimental Psychology: General, 131, 48-64.

WICKENS, C. D. (2002). Multiple resources and performance prediction. Theoretical Issues in Ergonomics Science, 3, 159-177.

\section{NOTE}

1. For an array with $N$ squares, the proportion in working memory can be estimated as $k / N$ whether the correct answer is same or different, and the proportion guessed correctly can be expressed as $(1-k / N) * g$ when the correct answer is different and $(1-k / N) *(1-g)$ when the correct answer is same. This leads to $p$ (hits) $=k / N+(1-k / N) * g$ and $p($ correct rejections $)=k / N+(1-k / N) *(1-g)$. Adding equations and rearranging terms, $k=N *$ (hits + correct rejections -1$)$. For experiments such as ours, in which the array changes on half of the trials, the formula simplifies to $k=N *[(2 *$ proportion correct $)-1]$. Rearranging terms, it can be shown that the proportion correct equals $k /(2 N)+.5$.

(Manuscript received December 20, 2002; revision accepted for publication April 21, 2003.) 